Article (refereed) - postprint

Tuoriniemi, Jani; Juergens, Monika D.; Hassellöv, Martin; Cornelis, Geert. 2017. Size dependence of silver nanoparticle removal in a wastewater treatment plant mesocosm measured by FAST single particle ICP-MS. Environmental Science: Nano, 4 (5). 1189-1197. 10.1039/C6ENO0650G

Copyright (C) The Royal Society of Chemistry 2017

This version available http://nora.nerc.ac.uk/516926/

NERC has developed NORA to enable users to access research outputs wholly or partially funded by NERC. Copyright and other rights for material on this site are retained by the rights owners. Users should read the terms and conditions of use of this material at http://nora.nerc.ac.uk/policies.html\#access

This document is the author's final manuscript version of the journal article following the peer review process. There may be some differences between this and the publisher's version. You are advised to consult the publisher's version if you wish to cite from this article.

http://www.rsc.org

Contact CEH NORA team at noraceh@ceh.ac.uk

The NERC and CEH trademarks and logos ('the Trademarks') are registered trademarks of NERC in the UK and other countries, and may not be used without the prior written consent of the Trademark owner. 


\title{
Size Dependence of Silver Nanoparticle Removal in a Wastewater Treatment Plant Mesocosm Measured by FAST Single Particle ICP-MS
}

\author{
Jani Tuoriniemi ${ }^{1}$, Monika D. Jürgens ${ }^{2}$, Martin Hassellöv ${ }^{3}$, Geert Cornelis ${ }^{1,4 *}$ \\ ${ }^{1}$ Department of Chemistry and Molecular Biology, University of Gothenburg, Gothenburg, \\ Sweden \\ ${ }^{2}$ Centre of Ecology and Hydrology (CEH), Wallingford, UK. \\ ${ }^{3}$ Department of Marine Sciences, University of Gothenburg, Gothenburg, Sweden \\ ${ }^{4}$ Department of soil and environment, Swedish University of Agricultural Sciences, Uppsala, \\ Sweden.
}

\begin{abstract}
The quantities of engineered nanoparticles (NP) released to the environment are often influenced by their fate in waste water treatment plants (WWTP). Here, $40 \mathrm{~nm}$ silver NP (AgNP) were spiked into a mesocosm simulating the process used at a major municipal WWTP. The evolution of the mass distributions and number concentrations were followed by fast acquisition speed technique single particle inductively coupled mass spectrometry (FAST spICP-MS) using a high-resolution ICP-MS. It was thus possible to detect smaller Ag containing NP than hitherto possible in similar studies. These small particles (ca. 5-10 nm in corresponding metallic $\mathrm{Ag}$ equivalent spherical diameter) were possibly dissolved $\mathrm{Ag}^{+}$ precipitated as $\mathrm{Ag}_{2} \mathrm{~S}$ particles. They were detected immediately upon spiking and were stable with respect to aggregation and thus much less removed by the WWTP process compared to the $40 \mathrm{~nm}$ AgNP. The results also suggested that any transformation of the latter AgNP occurred without dissolution. Most of these larger AgNP were probably removed by aggregation with large floc particles and subsequent sedimentation with the suspended particulate matter in the simulated WWTP process. The results have implications for differentiating the fate of nanoparticles as a function of size and demonstrate how spICP-MS can reveal such size-dependent fate dynamics.
\end{abstract}

\section{Environmental Significance Statement}

The size distributions of Ag nanoparticles during simulated waste water treatment were followed by FAST spICP-MS. The high sensitivity allowed to detect particles with an Ag mass as small as ca. $5 \mathrm{~nm}$ in corresponding metallic Ag equivalent spherical diameter. These small particles were contrary to expectations stable with respect to aggregation with suspended particulate matter.

\section{Introduction}

The rapidly developing nanotechnology market is leading to exposure of the environment to engineered nanoparticles (NPs). Use of silver NP (AgNP) as an antiseptic, for instance, has spawned a demand for both environmental monitoring, and toxicological tests of particulate silver. A decade of environmental risk-assessment-related research on NPs has shown that some standard procedures used for dissolved chemicals must be altered, and that some of these procedures are even irrelevant for assessing NP risk. The number-based NP size distribution (PSD), for instance, informs NP risk assessment much more than mass concentrations $\left(c_{m}\right)$ alone, because the size of single NPs or their aggregates determines their fate and thus bioavailability in the environment ${ }^{1}$. The number concentration $\left(c_{p}\right)$ and PSD are 
thus more relevant dose metrics than mass in this respect, because aggregation rates increase linearly with this metric ${ }^{2}$.

A key phase in the life cycle of AgNPs is wastewater treatment (WWT), because the interaction of NPs with sludge determines the extent by which these NP are emitted to aquatic compartments, or are carried with the sludge to be deposited on agricultural soils or to be incinerated $^{3}$. Several studies have reported that, on a mass basis, more than $95 \%$ of NPs become associated with sludge ${ }^{4}$, but risk assessments of NP also need to be informed on the form of NP, i.e. their size, composition or other properties that determine their hazard or fate in following environmental compartments. Chemical transformations of AgNP during WWT following oxidative dissolution have been relatively well studied. This process is, depending on the availability of ligands, followed by precipitation of $\mathrm{AgCl}$ or $\mathrm{Ag}_{2} \mathrm{~S} \mathrm{NPs}^{4}$, processes that, lower the hazard potential of $\mathrm{AgNP}^{5}$. The size of NP aggregates likely changes as well during this process. The high salt concentration in wastewaters may induce aggregation, whereas dissolved organic matter (DOM), such as humic and fulvic acids stabilizing smaller particles, can also be found in wastewater ${ }^{6}$, and dissolution of AgNP reduces their size ${ }^{7}$.

Studies on these types of fate dynamics are relatively scarce, probably because they require powerful analytical techniques that determine more parameters than $c_{m}$ alone in challenging environmental matrices and that are fast enough to monitor the fast changes of PSD. Moreover, the techniques have to be sensitive and selective, because the concentrations of engineered NPs in the environment, including WWT plants (WWTPs), are in the ng $\mathrm{L}^{-1}$ range $^{8,9}$, whereas the concentration of naturally occurring particles is many orders of magnitude higher and these particles sometimes contain similar elements as the NP of interest $^{10}$. Single-particle inductively coupled plasma mass spectrometry (spICP-MS) is currently the only technique where specific analysis of number-based PSDs of NPs in environmental samples is possible ${ }^{10}$. The ion clouds produced by individual NP or NP aggregates in the ICP plasma are detected at a fast rate, after which the mass per ion cloud can be calculated into corresponding spherical diameters $\left(d_{\text {part }}\right)$ assuming a particular composition of the NP and the number of ion clouds into $c_{p}{ }^{8}$. ICP-MS is element-specific and sensitive for $\mathrm{Ag}$ and can thus measure mass distributions of relatively small Ag containing NP in the presence of many other particles.

The Fast Acquisition Speed Technique (FAST) was used to extend the capabilities of spICPMS in this work. The presence of a dissolved background or carry-over can increase the size detection limit $\left(D L_{s}\right)$ of spICP-MS, because small particles generate similar signals to dissolved ions ${ }^{8}$. Conventional spICP-MS typically uses dwell times $\left(t_{\text {dwell }}\right)$, i.e. the time over which ion clouds are measured, in the order of a few ms. In FAST mode, the $t_{d w e l l}$ is even shorter, typically $30 \mu \mathrm{s}$, so that more than one data point per ion cloud is obtained. The authors previously developed an algorithm to distinguish dissolved ions from small nanoparticle events in FAST data, a procedure that can reduce the size detection limit ${ }^{11}$. Moreover, FAST has a more extended linear range in terms of particle number concentration $^{11,12}$ than conventional spICPMS, because especially at higher concentrations, more than one ion cloud formed by particles can arrive at the detector during the long $t_{d w e l l}$ used in conventional spICP-MS.

Using spICP-MS, NPs have previously been detected in freshwaters ${ }^{7}, 13$ and WWTP effluents $^{8,9}$, but the present study is the first to use spICP-MS for studying time-dependent 
processes of AgNP in a WWT mesocosm. The goal was to investigate the time-dependent changes in AgNP mass distribution during WWT. It is already established that, in general, most of AgNP are sulfidized and become aggregated and removed with the sludge. However, less is known about the size dependency of this process. With FAST spICP-MS there is potential to reach size detection limits $\left(D L_{s}\right)$ as low as $\sim 5 \mathrm{~nm}$ and therefore to probe at least part of the size range where the particles' physical properties change rapidly with size.

\section{Experimental}

Chemicals. A suspension of $40 \mathrm{~nm}$ nominal diameter citrate coated AgNP was obtained from BBI, UK. The dissolved Ag, In, and Au standards (Ultra Scientific, RI, USA) for spectrometer tuning and calibration were diluted in ultrapure water from $1000 \mathrm{ppm}$ solutions in $2 \% \mathrm{HNO}_{3}$. A certified $60 \mathrm{~nm}$ CRM Au NP suspension was purchased from NIST (Gaithersburg, MD, USA) for nebulization efficiency $\left(f_{\text {neb }}\right)$ determination.

\section{spICP-MS analysis and data treatment}

Calibration and particle characterization. The ICP-MS instrument tune parameters were optimized using dissolved In standards. The instrument tune and calibration parameters, and sample introduction components are given in Table 1 . The nebulization efficiency, $f_{n e b}$ was determined based on the NIST $60 \mathrm{~nm}$ Au NP according to the size method by Pace et al. ${ }^{14}$. The ICP-MS was first calibrated using dissolved Au standards followed by a measurement of $10^{6}$ times diluted (in water) dispersion of the $60 \mathrm{~nm} \mathrm{Au} \mathrm{NP}$. The assumed $f_{\text {neb }}$ was adjusted during calculations until the measured spICP-MS number-average $d_{\text {part }}$ of the NIST $60 \mathrm{~nm}$ $\mathrm{CRM} \mathrm{Au}$ particles matched the certified number-average based diameters, as measured by Electrospray Scanning Mobility Particle Sizer, SEM, and TEM. For the measurement of $\operatorname{AgNP} c_{p}$ and PSD the ICP-MS sensitivity was calibrated using dissolved Ag standards (0.03, $0.1,1,2,5$, and $10 \mu \mathrm{g} \mathrm{L}^{-1}$ ) that were measured at the same measurement settings in water or WWTP liquor (Table 1). A Burgener nebulizer ${ }^{15}$ was used, a nebulizer that is adapted for introducing particles into an ICP-MS ${ }^{16}$.

Data acquisition. All measurements were carried out on an Element 2 (Thermo electron, Bremen, Germany) sector field ICPMS instrument. A FAST data acquisition method ${ }^{11}$ with the $t_{d w e l l}$ set to $0.1 \mathrm{~ms}$ was used for all measurements of AgNP samples. Calibrations were carried out using a $10 \mathrm{~ms} t_{d w e l l}$. For each sample $300000-350000$ dwells were acquired to monitor the signal during at least $30 \mathrm{~s}$.

Data analysis. The data was processed in matlab 7.11 software. A data treatment algorithm described elsewhere ${ }^{11}$ was used to distinguish peaks due to NP from the dissolved analyte signal. In short, the algorithm is an adaption of the iterative outlier detection algorithm ${ }^{8}$ for datasets generated by rapid data acquisition, were the particle events may be spread over several dwells. Its parameters were optimized using the same AgNP colloid and instrumentation as in the study by Tuoriniemi et al. ${ }^{11}$. The algorithm identifies peaks by locating zero crossings of the derivative (i.e. where the derivative (slope) changes from positive to negative) and requiring the intensity at the same time to exceed a predefined number $(n=5)$ of standard deviations of the dissolved background signal. The peak dwell, and a set number of dwells $(w=3)$ surrounding it are considered as part of the particle event and removed from the dataset. This procedure is repeated until no more peaks are detected. If the dissolved background signal is low enough for the detection threshold to fall below 2 ion 
counts, the algorithm resorts to cluster detection for identifying the smallest particles. The criterion for detection is that at least $M=4$ ions should arrive in patterns characteristic for Gaussian particle events. Although it was previously shown that $M=2$ can be sufficient for distinguishing particles with diameters close to the $D L_{s}$, a higher value of 4 was used in this study to minimize the frequency of false positives.

To summarize, the ions arriving during the $\pm w$ dwells surrounding an identified particle event are assigned as particle bound $\mathrm{Ag}$, while ions that cannot be associated with particles are considered to originate from dissolved Ag. Because dissolved Ag arrives simultaneously with particles, the average dissolved Ag signal is subtracted from the particle bound Ag signal intensities. The particle-bound intensities are thus integrated per particle event and the integrated intensity is calculated, using the calibrated nebulization efficiency into a corresponding spherical diameter, i.e. a spherical shape is assumed for calculating diameters from the $\mathrm{Ag}$ mass per particle event. Note that both $\mathrm{Ag}^{0}$ and e.g. $\mathrm{Ag}_{2} \mathrm{~S}$ composition can be assumed during this calculation as single-element spICP-MS does not provide info on the composition of each particle.

Table 1 Typical values for ICP-MS tune and calibration parameters

\begin{tabular}{|c|c|}
\hline Sample gas flow rate & $0.520 \mathrm{ml} \mathrm{min}-1$ \\
\hline Auxiliary gas flow rate & $0.75 \mathrm{ml} \mathrm{min}^{-1}$ \\
\hline Cool gas flow rate & $15.01 \mathrm{ml} \mathrm{min}^{-1}$ \\
\hline Sample uptake rate & $0.48 \mathrm{ml} \mathrm{min}^{-1}$ \\
\hline RF power & $1205 \mathrm{~W}$ \\
\hline Nebulizer & Burgener Mira mist \\
\hline Spray chamber & $4 \mathrm{~mL}$ Cyclonic (Glass expansion) \\
\hline Torch & Fassel type (Thermo Finnigan) \\
\hline Sample cone & Nickel $1.0 \mathrm{~mm}$ orifice diameter \\
\hline Skimmer cone & Nickel $0.8 \mathrm{~mm}$ orifice diameter \\
\hline Sensitivity & $4.6^{*} 10^{18}$ (counts $\mathrm{g}^{-1} \mathrm{Ag}$ in pure water) \\
\hline Nebulization efficiency & $0.88 \%$ \\
\hline
\end{tabular}

\section{Waste water treatment plant mesocosm}


Waste water treatment process. The secondary treatment process used at the GRYAAB ${ }^{17}$ WWTP in Gothenburg was emulated on a laboratory scale by letting primary treated sewage spiked with AgNP pass through a mesocosm where the conditions were as close as possible to subsequently the anoxic, oxic, and settling stages used at the secondary treatment of the plant.

The process at the plant can be outlined as follows. After coarse filtering of the raw wastewater and sedimentation of large debris in the primary treatment, iron sulfate is added as a flocculating agent. The primary treated wastewater is mixed with recirculated activated sludge (RAS) of the secondary treatment and recirculated effluent from a nitrification trickling filter. This mix then undergoes anoxic, and then oxic conditions where air is pumped into the reactor achieving $c a .2 .5 \mathrm{mg} \mathrm{L}^{-1}$ of dissolved oxygen. This is followed by a settling stage where the flocks are allowed to settle and the cleared effluent flows to further treatment (nitrification, phosphate removal, denitrification and filtering) and the settled sludge is either recirculated to the oxic treatment as RAS or removed for further treatment and eventually disposed - for example to be used as fertilizer.

\section{WWTP Sampling.}

Because the mesocosm set up here is not a continuous system where sludge is recycled it requires realistic RAS. It is nearly impossible to produce RAS that bears any resemblance to that produced at an actual WWTP. Therefore it was chosen to sample the relevant fractions, primary treated wastewater, nitrification effluent, and RAS from GRYAAB and mix them in a mesocosm. A disadvantage is that the WWT process cannot be finely regulated to e.g. investigate specific chemical influences such as $\mathrm{pH}$ and ionic strength, i.e. the composition of the WWTP fractions was a given. This choice may limit the potential to generalize from the current study to other WWTPs.

Five experiments were carried out in which the liquor for the microcosm experiment was created by mixing outflow from the primary wastewater treatment, RAS, recirculated nitrification trickling filter outflow and iron sulfate in the same proportions as at the WWTP achieving a TSS as in the secondary treatment at the plant at the day of sampling. It was chosen not to sample RAS from the outflow of the secondary clarifier, because preliminary experiments showed that the settled sludge was much less active compared to sludge suspension taken before the clarifier. So, the final settling stage was simulated in the laboratory in an Imhoff cone before the beginning of the experiment and sludge was collected from the bottom of the cone to achieve the TSS concentration that was measured in RAS of the plant at the day of sampling. This allowed the simulated RAS to be fresher and therefore more realistic than if already thickened RAS had been collected and then stored for the time needed to return to the laboratory and set up the experiment. The high biological activity of sewage sludge means that sample characteristics can change significantly in a short period of time. Therefore, care was taken to keep sample storage to a minimum and all sampling occurred less than 2 hours before the start of the spICP-MS measurements. To protect the ICP-MS from large fibers coming mainly from toilet paper the WWTP samples were filtered through a $0.1 \mathrm{~mm}$ mesh prior to the settling stage described above.

Spiking of AgNP. The $40 \mathrm{~nm}$ AgNP stock suspension was sonicated to break up any clusters of NP and spiked to a concentration of 5, or $50 \mu \mathrm{g} \mathrm{L}^{-1}$ into the WWT mixture at the beginning of the experiment. The anoxic stage was simulated by gently stirring the mixture in a glass beaker for 180 minutes while measuring dissolved oxygen using an oxygen probe. The 
sample was then transferred to a plastic Imhoff cone for 120 minutes of oxic treatment. Here, air was pumped into the sample while continuously measuring dissolved oxygen using a probe, to maintain a dissolved oxygen concentration of $\sim 2.5 \mathrm{mg} \mathrm{L}^{-1}$, which is the operating oxygen concentration at the WWTP. Finally, the air was turned off and the sample was allowed to settle for 100 minutes in the cone, simulating the clarifier stage. The following parameters were monitored during these experiments: temperature, reactive phosphate, nitrate, nitrite, bromide and chloride, $\mathrm{pH}$, conductivity, and the dissolved oxygen concentration. Phosphate was measured with the molybdenum blue method, and the anions were measured using ion chromatography. The $\mathrm{pH}$, conductivity and dissolved oxygen were measured using electrodes.

SpICP-MS in WWTP liquor. High rates of carbon deposition on the sampler cone lead to a gradually decreasing sensitivity during the mesocosm experiments. Ag standards were therefore measured in pure water between the wastewater samples to correct for sensitivity drift which was calculated by linear interpolation. Experiment 1 was conducted with a spiked AgNP concentration of $5 \mu \mathrm{g} \mathrm{L}^{-1}$ and the sensitivity calibration was done with $\mathrm{Ag}$ standards dissolved in pure water. The procedure was changed for experiments 2 and 3 , because the measurements failed several times due to the high solids deposition rate from the concentrated WWTP liquor. A higher concentration of AgNP $\left(50 \mu \mathrm{g} \mathrm{L}^{-1}\right)$ was used so that the mesocosm samples could be diluted 5 times immediately before measurement to lower the TSS concentration. It was not an option to only dilute 5 times without increasing the AgNP dose, because the sensitivity would not have been high enough to measure a statistically significant particle number within the time constraints of the ongoing mesocosm experiment that is producing samples continuously. For experiments 2, and 3 the Ag sensitivity calibration was carried out in the 5 times diluted WWTP liquor instead of pure water, but the correction for sensitivity drift was still carried out in pure water because the properties of the WWTP liquor change during the experiment.

\section{Results and discussion}

Chemical parameters. Some monitored chemical parameters are shown in Table 2. Other measured parameters $\left(\left[\mathrm{O}_{2}\right]\right.$, Reactive phosphate, $\left.\left[\mathrm{Br}^{-}\right],\left[\mathrm{F}^{-}\right],\left[\mathrm{NO}_{3}{ }^{2-}\right],\left[\mathrm{NO}_{2}{ }^{2-}\right],\left[\mathrm{Cl}^{-}\right],\left[\mathrm{SO}_{4}{ }^{2-}\right]\right)$ are shown in supporting information (SI). The properties of the WWTP mixture in terms of $\mathrm{pH}$, conductivity, and chemistry were similar for all three experiments, except for the TSS, which was considerably lower during the first experiment. This was because the RAS flow at the WWTP into the anoxic stage, that provides most of the TSS, was considerably lower than normal at the time of sampling.

The oxygen content dropped to ca. $0.1 \mathrm{mg} \mathrm{L}^{-1}$ almost immediately during the anoxic stage in experiments 2, and 3, whereas it took ca. $30 \mathrm{~min}$ in experiment 1 to decrease to such levels from the initial $5.5 \mathrm{mg} \mathrm{O}_{2} \mathrm{~L}^{-1}(\mathrm{SI})$. This was probably because of the relatively low TSS. The oxygen level was kept stable around $2.5 \mathrm{mg} \mathrm{L}^{-1}$ during the anoxic treatment by adjusting the air flow through the suspension, whereas the oxygen concentration decreased again during the settling stage, albeit slower than during the anoxic stage (SI).

The reactive phosphate concentration decreased, and most of the nitrite and all nitrate was depleted during the anoxic stage. This indicates that the microcosm was microbially active throughout all experiments with microorganisms using nitrate as electron acceptor and using phosphate to grow. The explanation for the higher $\mathrm{Br}^{-}$to $\mathrm{Cl}^{-}$ratios in experiments 2, and 3 is 
probably the higher RAS content that is enriched in $\mathrm{Br}^{-}$released from decomposed biological matter $^{18}$. In summary, it can be argued that the microcosm experiments demonstrated chemical conditions similar to actual plants.

Table 2 Chemical parameters at the start of the microcosm experiments in undiluted mixed WWTP samples.

\begin{tabular}{lllll}
\hline Experiment & $\begin{array}{l}\text { Spiked Ag } \\
\text { concentration } \\
\left(\boldsymbol{\mu g ~ \mathbf { L } ^ { - 1 } )}\right.\end{array}$ & $\begin{array}{l}\text { Conductivity } \\
\left(\boldsymbol{\mu S \mathbf { c m } ^ { - 1 } )}\right.\end{array}$ & $\mathbf{p H}$ & $\begin{array}{l}\text { TSS } \\
(\%)\end{array}$ \\
\hline $\mathbf{1}$ & 5 & 900 & 7.3 & 0.7 \\
$\mathbf{2}$ & 50 & 1050 & 7.1 & 2.4 \\
$\mathbf{3}$ & 50 & 1060 & 7.0 & 2.3 \\
\hline
\end{tabular}

Sensitivity and size detection limit. The $D L_{s}$ for AgNP detection as a function of time during experiments 1, 2, and 3 are shown in Fig. 1. The decreasing sensitivity clearly had a profound effect on the $D L_{s}$. In conventional spICP-MS, the $D L_{s}$ is often determined by the dissolved background $^{8}$. Here the $t_{d w e l l}$ was much lower, and the dissolved signal was therefore low enough for the $D L_{s}$ to be determined by the sensitivity of the instrument. The $D L_{s}$ in the current study is the $d_{\text {part }}$ of a large enough particle to deliver 4 ions on average to the detector, so that the particle event can be recognized as a Gaussian cluster. The sensitivity was considerably higher in experiment $1\left(D L_{s} \sim 5.4 \mathrm{~nm}\right)$ and fluctuated less as a function of time compared to the two other experiments $(D L s \sim 30-40 \mathrm{~nm})$ despite the 5 times dilution bringing the TSS down to similar levels to experiment 1 . The main reason for this was deposition of solid material on the sampler cones. Despite the correction for drift, the accuracy in determined sizes and concentrations for experiment 2, 3 were most likely reduced compared with experiment 1 . These experiments were terminated when the sensitivity deteriorated after $>200 \mathrm{~min}$, because the $D L_{s}$ became very high and very few Ag particles could be detected any more (Fig. 1).

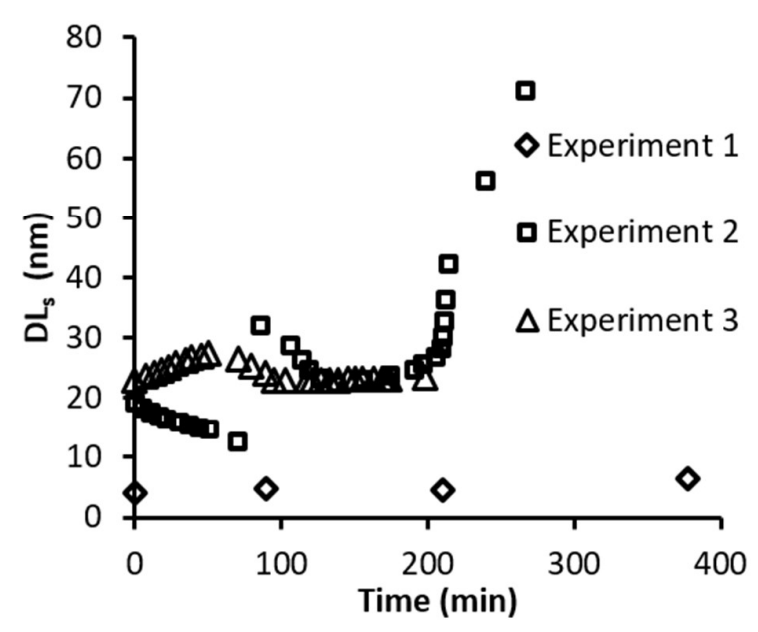

Fig. 1. The $D L_{s}$ during the mesocosm experiments.

The sensitivity of an ICP-MS is proportional to the product $f_{\text {neb }} * f_{\text {ion }} * f_{\text {trans }}$, with $f_{\text {ion }}$ being the fraction of analyte converted to singly charged ions in the plasma, and $f_{\text {trans }}$ giving the fraction 
of these that eventually reach the detector ${ }^{8}$. In conventional ICP-MS, it is often enough to calibrate for sensitivity in the same matrix as the samples to compensate for matrix effects. However, to calculate accurate values of $c_{p}$ and $d_{\text {part }}$ with $\mathrm{spICP}-\mathrm{MS}^{8}$ one would need to determine both $f_{\text {neb }}$ and $f_{\text {ion }} * f_{\text {trans }}$ in the same matrix, and then separately monitor the drift in these parameters to accurately calculate the corresponding spherical size of the NPs at all measurements times.

Organic loadings. The high organic loadings in the WWT samples may have increased or decreased $f_{n e b}$ and $f_{\text {ion }}{ }^{19}$. Surface tension is the main parameter controlling $f_{\text {neb }}$ of solutions of non-volatile organic compounds ${ }^{19}$. The WWTP liquor contains surfactants and the resulting lower surface tension might improve $f_{n e b}{ }^{19}$, but at the same time the particles that are large enough to be removed in the spray-chamber are wetted and may reduce the $f_{\text {neb }}$ by taking water with them to the spray-chamber waste flow ${ }^{20}$. A high organic load requires increased plasma energy to break the organic compounds. This effectively quenches the plasma, reducing $f_{\text {ion }}$, and more so if the organic loading is higher ${ }^{21}$. While the $f_{\text {ion }}$ and $f_{\text {neb }}$ are thus both relatively sensitive to organic loading, $f_{\text {trans }}$ is most likely affected much less. Liu and Beauchemin $^{21}$ found that whereas the intensity of ions in the presence of organic solvents was lower compared to $1 \%$ nitric acid as a solvent, only addition of solvents with low boiling points resulted in a different spatial radial profile of ions generated in the plasma, because they evaporate earlier from the water. Ag ions measured in WWTP samples thus probably had the same radial profile compared to $\mathrm{Ag}$ in clean water and thus a similar $f_{\text {trans }}$, albeit that a lower proportion was ionized. The $f_{\text {trans }}$ will however decrease when sampler cone openings are narrowed by deposition of solids.

Because $f_{\text {neb }}$ was too low to be measured by the waste collection method it was assumed in all experiments that its value was equal to that in pure water. If its value in WWTP liquor was for instance smaller, the $c_{p}$ would be underestimated. The sensitivity calibration for experiments 2 , and 3 was done in WWTP liquor diluted to the same concentration as the samples. The Ag sensitivity was $20-30 \%$ of that in pure water. By matrix calibrating regularly, the decrease in sensitivity can be compensated for, but the $c_{p}$ and $d_{p a r t}$ can still not be calculated with full certainty, because the contributions of $f_{n e b}$, $f_{\text {ion, }}$, and $f_{\text {trans }}$ cannot be distinguished from each other. However, the matrix calibration still served the purpose of establishing boundaries for the expectable magnitude of errors. For instance, any error in $f_{\text {ion }} * f_{\text {trans }}$ is propagated as their third root to the $d_{\text {part }}^{8}$. Therefore, it is unlikely that ignoring the $80-70 \%$ drop in sensitivity in waste water would ever cause the $d_{\text {part }}$ to appear more than 30-40\% smaller than their actual size, even in the worst case when $f_{n e b}$ equals to its value in pure water. The regular measurement of Ag standards during the mesocosm experiments was most likely enough to compensate for the drift in $f_{\text {trans }}$ due to sampler cone blockage, because the buildup of contamination occurs on slower time scales than the measurement of a single sample.

Evolution of the AgNP size distribution. Fig. 2 shows the evolution of the distribution of the corresponding equivalent spherical diameter (PSD) during experiment 1. Even though spICPMS measures the mass of Ag per particle, the PSD provides are more intuitive way of representing spICPMS results. Such a representation requires an assumption on the shape and composition of the particles. In this case, spherical metallic silver was used, but implications of other compositions are discussed further. The PSD measured in ultrapure water prior to spiking is broad and has a modal value close to its nominal average diameter of $40 \mathrm{~nm}$. There is a tail in the PSD that disappeared upon spiking to the mesocosm. This tail thus most likely 
contained loose agglomerates that were re-dispersed upon spiking in the WWT liquor, because FAST spICP-MS has nearly no artefacts of double particle events, which in conventional spICP-MS could for the measured $c_{p}$ cause tails in the PSD at larger particle diameters ${ }^{11-12}$. It can be calculated that the corresponding spherical diameter of aggregates consisting of $n$ particles each having diameter $d$ equals $n^{1 / 3} d_{\text {part }}$. The corresponding spherical diameters of doublet or triplet aggregates of $40 \mathrm{~nm}$ are thus 50.4 and $57.7 \mathrm{~nm}$ respectively. The tail of particles at ca. $50 \mathrm{~nm}$ in pure water therefore suggests that the stock dispersion initially contained doublets, which were re-dispersed in the WWTP liquor. The doublets were possibly loosely aggregated and were re-dispersed because of shear and/or the presence of stabilizing dissolved organic matter (DOM).

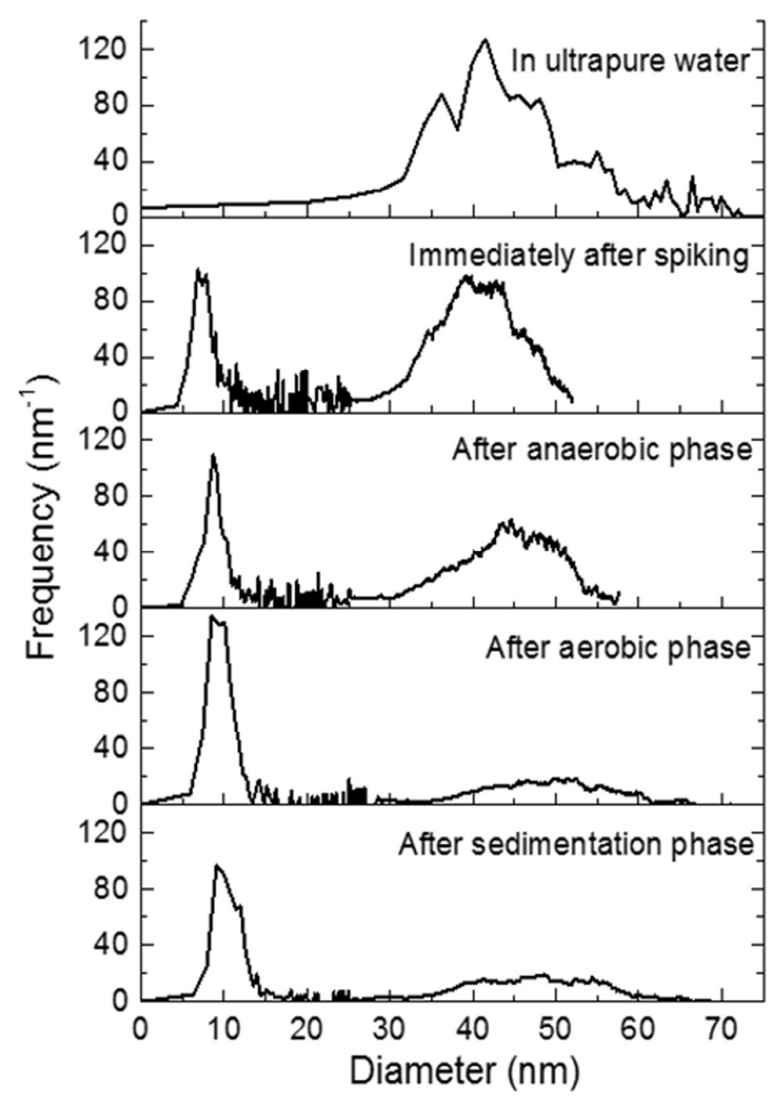

Fig. 2. Evolution of the AgNP size distribution during experiment 1.

The modal diameter of the $40 \mathrm{~nm}$ particles did not decrease during the mesocosm experiments but stayed close to its value measured in pure water (i.e. $40 \mathrm{~nm}$ ). This indicates that matrix effects did not significantly affect the spICPMS measurements. The tail of large particles found in the spiking suspension returns as the AgNP re-aggregate during the WWT process. Note that doublets cannot be distinguished from two individual particles hetero-aggregated on the same sludge particle, not even in FAST spICP-MS. Fig. 2 therefore suggests heteroaggregation with AgNP gathering on the same sludge particle, because homo-aggregation of AgNP is unlikely given the relatively low AgNP concentration added to the WWT liquor, whereas attachment rates of NPs to WWT sludge are known to be high ${ }^{22}$. The PSD fell partially below the $D L_{s}$ in experiment 2 and 3 . Furthermore, the $c_{p}$ are accurate only for $d_{\text {part }}$ significantly exceeding the $D L_{s}$. This is both because the relative number of false positives 
increases for particle sizes close to $D L_{s}$ and the probability for a particle that enters the plasma to produce a detectable event decreases ${ }^{11}$.

It is unlikely that the $40 \mathrm{~nm}$ AgNP were still pristine AgNP throughout the WWT process. Several studies have emphasized fast sulfidation kinetics of AgNP during a WWT process ${ }^{23}$, a process that has been observed to occur without prior dissolution of the $\mathrm{AgNP}^{24-26}$. The mass of silver per particle thus remains the same which explains why the modal diameter does not decrease. The $\mathrm{HS}^{-}$concentration during the mesocosm experiments was not known. However, it is plausible that sulfidation did occur given that the chemical parameters measured were representative and that there were ongoing microbial degradation of the waste producing HS'.

Fig. 3 shows the evolution of the modal value of the second peak at 5-10 nm that started to form almost immediately upon spiking AgNP into the WWT liquor and then grew in modal diameter during the experiment. Note, again, that spICP-MS measures corresponding spherical diameters calculated from the total Ag mass that arrives simultaneously. Such small AgNP can therefore possibly originate from dissolved Ag adsorbed to relatively large suspended organic particles. A simple calculation (see SI) based on the density of adsorption sites for metal ions ${ }^{27}$ suggests, however, that this is unlikely.

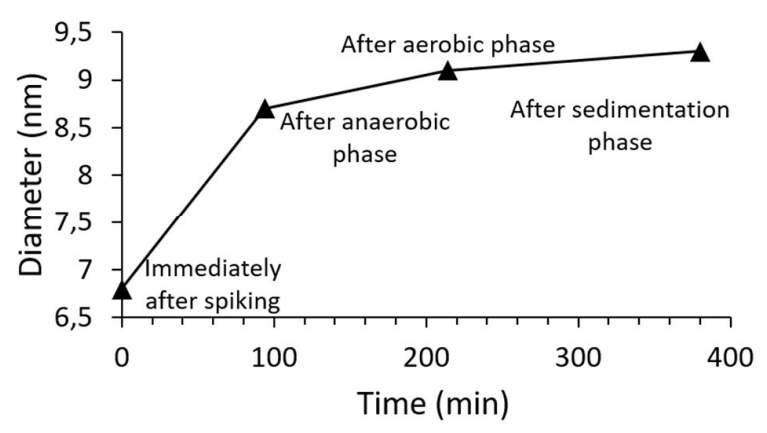

Fig. 3. The evolution of the modal metallic Ag equivalent spherical diameter of small NP during the WWTP process.

The small peak therefore originated more likely from $\mathrm{Ag}_{2} \mathrm{~S}$, or alternatively $\mathrm{AgCl}$ particles, that precipitated from dissolved $\mathrm{Ag}$. The equivalent spherical diameters of $\mathrm{Ag}_{2} \mathrm{~S}$ and $\mathrm{AgCl}$ would be $\sim 8 \%$ and $\sim 12 \%$ larger than metallic $\mathrm{Ag}$, which is within the range of uncertainty in these measurements and does not alter the conclusions that could be drawn from Figs. 2, and 3. A fraction of the Ag particles in a AgNP colloid always occurs as dissolved Ag that can precipitate very fast with sulfide ions during WWT. Note that, although significant in number, this peak did never contain more than $2 \%$ of the total mass of Ag.

Figs. 2, and 3 shows that the freshly formed AgNP remain almost constant in number throughout the experiment, whereas the $40 \mathrm{~nm}$ AgNP (Figure 2) clearly decrease in number over time. There appeared to be loss of the latter particles during all three stages (anoxic, oxic and clarifier). The spICP-MS cannot show whether the AgNP are associated with sludge or not, but most likely they were, because all previous studies on AgNP fate in WWTP have shown a fast partitioning of AgNP to sludge particles ${ }^{28}$. What has not been studied, though, is whether the attachment rate between sludge particles and AgNP depends on size. This relation is not only poorly understood for the case of sludge. Higher attachment rates are theoretically expected for smaller particles ${ }^{29-30}$, but experiments with algae ${ }^{31}$ or deposition in heterogeneous media ${ }^{32-33}$ have shown that small particles are attaching less efficiently 
compared to larger NPs, a phenomenon that has been attributed to the occurrence of a secondary energy minimum or, that smaller nanoparticles are assumed to be more sensitive to surface roughness and heterogeneities. Another explanation for the unexpected stability comes from assuming that the flocs surfaces are best described as a mesh of polymeric strands rather than hard spheres. Larger particles can, because of their dimensions, interact with more strands and binding sites. The association with the flocs by multiple bonds becomes stronger in a way analogous to the chelate effect ${ }^{34}$ upon complexation of metal ions. In any case, the interactions between NP and waste water flocs requires further investigation as it bears relevance to the subsequent fate of engineered NP.

Evolution of AgNP concentration over time. Fig. 4 shows the $c_{p}$ and $c_{m}$ of particle bound Ag during the mesocosm experiments. The scatter in data is significant. A statistically significant number of particles ${ }^{35}$ were detected in most of the measurements so the scatter was probably caused by the complex heterogeneous matrix. During a typical measurement $\sim 0.5$ $\mathrm{mL}$ of sample is nebulized of which only $\sim 2.5 \mu \mathrm{L}$ enters the plasma. The precision of graphite furnace atomic absorption spectrometry was found to be dependent on the sample mass for such small volumes of slurry samples ${ }^{36}$. The same is probably also true for ICP-MS spectrometers. For experiments 2 , and 3 the fluctuating $D L_{s}$ also overlaps with the PSD.
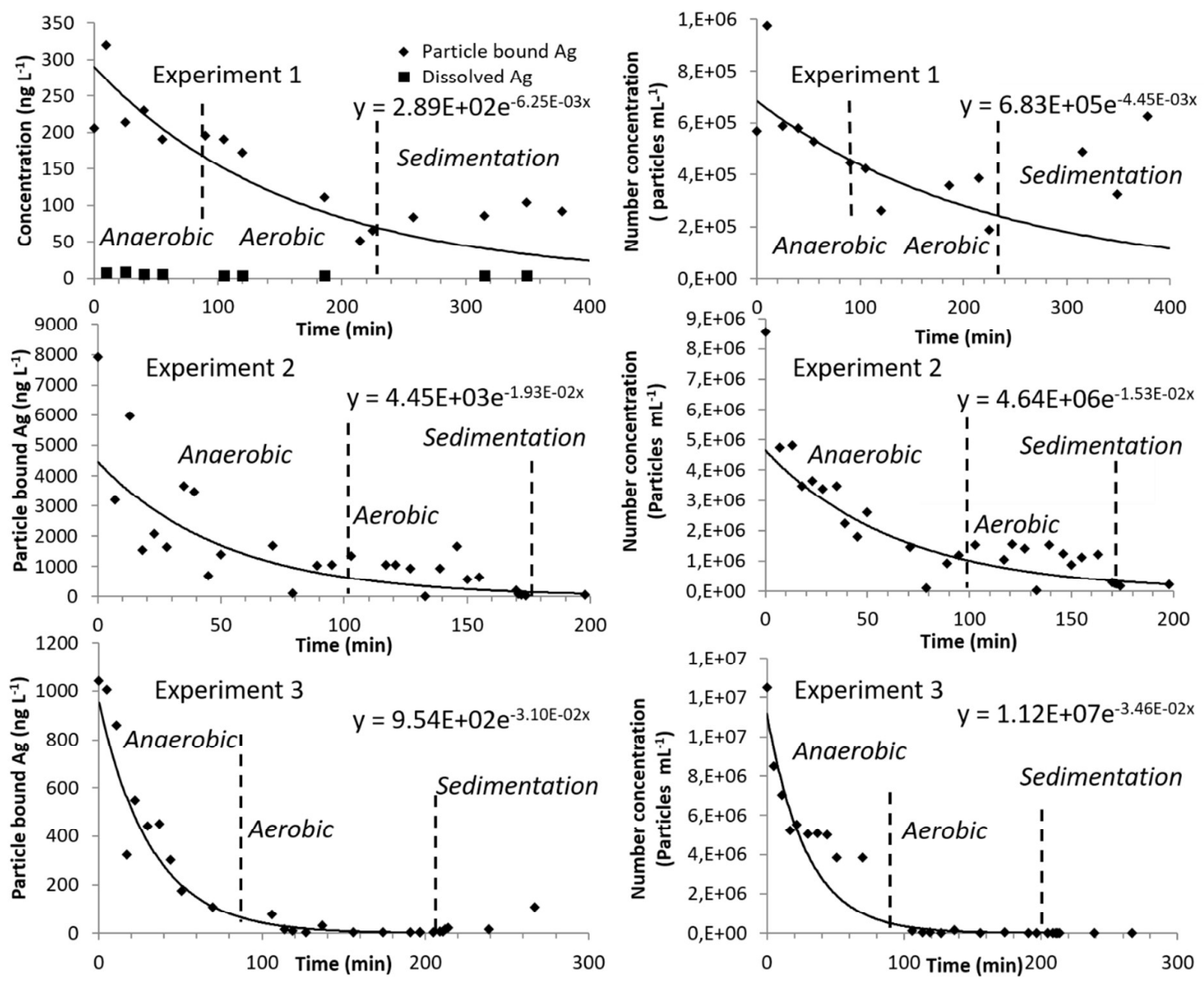

Fig. 4. The concentrations of AgNP during the mesocosm experiments fitted with an exponential decay. 
During experiment 1 , the $c_{m}$ decreased by 100-200 ng L $\mathrm{L}^{-1}$ over the course of the experiment (Fig. 4). As discussed earlier, there were no indications that the AgNP had dissolved, and such a loss in particle bound $\mathrm{Ag}$ would have been matched by a corresponding increase in the dissolved concentration. Instead the $c_{m}$ of the dissolved fraction including also the population of small AgNP remains at levels $<15 \mathrm{ng} \mathrm{L}^{-1}$. Furthermore, because the $c_{p}$ of NP measured by spICP-MS do agree with reference values obtained by other methods, ${ }^{35}$ adsorption on container walls are unlikely to play any significant role on the timescale of the experiment. The missing particles therefore, as argued earlier, aggregated with flocks and escaped detection by being too large to pass the spray chamber.

Note that the decreasing concentrations cannot be only an artefact of lowered $f_{\text {neb }}$ or $f_{\text {ion }}$ because in such case, the modal $d_{\text {part }}$ would decrease with $f_{\text {ion }}$, and the $f_{\text {neb }}$ and concentrations would probably recover when the TSS drops during the sedimentation stage, but none of this is observed.

Because of the scatter in data, the trends are easier to visualize if the concentrations are fitted with a suitable function. The size distribution of flocs in a given shear field and TSS concentration attains a steady-state condition following a transitory period of attachment and detachment, after which the size distribution is relatively stable over time ${ }^{37}$. Because the $c_{p}$ of suspended particulate matter in the WWTP liquor is much higher than that of the spiked $\mathrm{AgNP}$, it is possible that the aggregation of AgNP to flocs too large to pass the sample introduction system is best described by first order kinetics. This would result in an exponential decay of the $c_{p}$, and $c_{m}$. Such functions were fitted in Fig. 4 to the concentrations during the anoxic and oxic stages. The concentrations at the sedimentation stage were not considered during the fitting, because contrary to the anoxic and oxic stage, the concentration of TSS and Ag attached to them decreased because of the additional process of sedimentation. The exponential functions described the trends relatively well. The half-lifes of the $c_{p}$ and $c_{m}$ of AgNP calculated from the fitted exponentials are shown as a function of TSS in Fig. 5. It seems that the AgNP were removed faster in experiments 2 and 3, because of the higher concentration of flocs, and because flocs tend to be larger at high TSS and therefore less able to pass the spraychamber ${ }^{37}$. The half-lives measured for the similar experiments 2 and 3 are relatively close to each other.

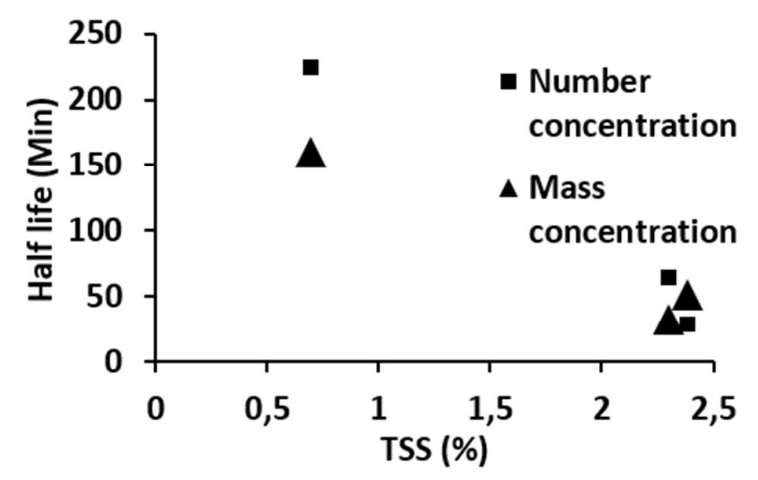

Fig. 5. The half-lives of the AgNP as a function of TSS.

\section{Conclusions}


The fate of AgNP was studied by FAST spICP-MS in mesocosms simulating a WWTP process. To our knowledge, spICP-MS measurements have not been conducted in such demanding matrices before, and this study gives therefore an account of the difficulties and practical considerations necessary for characterizing samples with a high solids content. It was possible to obtain information on the mass distribution, and the $c_{p}$ and $c_{m}$ of AgNP that were free or aggregated with flocs small enough to pass the spray chamber. Measuring the mass distribution that gives an indication of the dimensions of $\mathrm{Ag}$ containing particles, can complement earlier ${ }^{4}$ WWTP fate studies focusing on mass concentrations and speciation. The particles were not dissolving, which is in accordance with earlier studies suggesting that the particles are sulfidized directly instead of a dissolution/reprecipitation mechanism. Most of the AgNP probably aggregated with flocs, agreeing with earlier studies concluding that AgNP tend to partition to the sludge. However, this mechanism appeared size-dependent as there were $<10 \mathrm{~nm}$ particles that may have partitioned less efficiently to flocs during the experiments. Relatively smaller NP are thus potentially removed less efficiently compared to relatively larger particles, which should be confirmed in further experiments as it has implications for the fate of NPs that travel through WWT during their environmental lifetime. The fate of engineered NP released into natural water systems after passing or circumventing WWTP is also governed by heteroaggregation and subsequent sedimentation with the native particle population ${ }^{38}$. Small particles that are highly stable with respect to aggregation are therefore at risk of being somewhat more persistent in the environment.

Acknowledgements The authors would like to acknowledge funding sources from the European framework program projects NANOFATE (CP-FP 247739), MARINA (CP-FP 263215), GuideNANO (CP-FP 604387), The Swedish Research council (CC0393701) for supporting this work.

\section{References}

1. G. Cornelis, K. M. Hund-Rinke, T. Kuhlbusch, N. Van den Brink, C. Nickel, Fate and bioavailability of engineered nanoparticles in soils: a review, Crit. Rev. Env. Sci. Technol., 2014, 44, 2720-2764.

2. M. Elimelech, J. Gregor, X. Jia, R.A.F. Williams, Particle Deposition and Aggregation: Measurement, Modeling, and Simulation, Elsevier, Amsterdam, the Netherlands, 1995.

3. F. Gottschalk, B. Nowack, The release of engineered nanomaterials to the environment, J. Environ. Monit., 2011, 13, 1145-1155.

$4 . \quad$ R. Kaegi, A. Voegelin, B Sinnet,S Zuleeg, H. Hagendorfer, M. Burkhardt, H. Siegrist, Behavior of metallic silver nanoparticles in a pilot wastewater treatment plant, Environ. Sci. Tech., 2011, 45, 3902-3908.

5. C. Levard, E. M. Hotze, B. P. Colman, A. L. Dale, L. Truong, X. Y. Yang, A. J. Bone, G. E. Brown Jr.,R. L. Tanguay, R. T. Di Giulio, E. S. Bernhardt, J. N. Meyer, M. R. Wiesner, G. V. Lowry, Sulfidation of Silver Nanoparticles: Natural Antidote to Their Toxicity, Environ. Sci. Tech., 2013, 47, 13440-13448.

6. M. A. Kiser, D. Ladner, K. D. Hristovski, P. Westerhoff, Nanomaterial transformation and association with fresh and freeze-dried wastewater activated sludge: Implications for testing protocol and environmental fate, Environ. Sci. Tech., 2012, 46, 70467053.

7. D. M. Mitrano, J. F. Ranville, A. Bednar, K. Kazor, A. S. Hering, C. P. Higgins, Tracking dissolution of silver nanoparticles at environmentally relevant 
concentrations in laboratory, natural, and processed waters using single particle ICP-MS (spICP-MS), Environ. Sci.: Nano, 2014, 1, 248-259.

8. J. Tuoriniemi, G. Cornelis, M. Hassellöv, Size discrimination and detection capabilities of single-particle ICP-MS for environmental analysis of silver nanoparticles, Anal. Chem., 2012, 29, 743-752.

$9 . \quad$ D. M. Mitrano, E. K. Lesher, A. Bednar, J. Monserud, C. P. Higgins, J. F. Ranville, Detecting nanoparticulate silver using single-particle inductively coupled plasmamass spectrometry, Environ. Tox. and Chem., 2012, 31, 115-121.

10. B. Nowack, M. Baalousha, N. Bornhöft, Q. Chaudry, G. Cornelis, J. Cotterill, A. Gondikas, M. Hassellöv, J. Lead, D. Mitrano, F. von der Kammer, T. Wontner-Smith, Progress towards the validation of modeled environmental concentrations of engineered nanomaterials by analytical measurements, Environ. Sci.: Nano, 2015, 2, 421-428.

11. J. Tuoriniemi, G. Cornelis, M. Hassellov, A new peak recognition algorithm for detection of ultra-small nano-particles by single particle ICP-MS using rapid time resolved data acquisition on a sector-field mass spectrometer, J. of Anal. At. Spectrom., 2015, 30, 1723-1729.

12. M. D. Montano, H. R. Badiei, S. Bazargan, J. F. Ranville, Improvements in the detection and characterization of engineered nanoparticles using spICP-MS with microsecond dwell times, Environ. Sci: Nano, 2014, 1, 338-346.

13. A. P. Gondikas, F. von der Kammer, R. B. Reed, S. Wagner, J. F. Ranville, T. Hofmann, Release of TiO2 Nanoparticles from Sunscreens into Surface Waters: A One-Year Survey at the Old Danube Recreational Lake, Environ. Sci. Tech., 2014, 48, 5415-5422.

14. H. E. Pace, N. J. Rogers, C. Jarolimek, V. A. Coleman, C. P. Higgins, J. F. Ranville, Determining transport efficiency for the purpose of counting and sizing nanoparticles via single particle inductively coupled plasma-mass spectrometry, Anal. chem., 2011,83, 93619369.

15. J.A. Burgener, US Pat., 5411 208, 1995.

16. A. Montaser, M. G. Minnich, J. A. McLean, H. Liu, in Inductively coupled plasma mass spectrometry, ed. A. Montaser, Wiley, New York (USA), 1:st edition, 1998, 83-264.

17. Information about the GRYAAB plant and the waste water treatment process, http://www.gryaab.se/om-gryaab/publikationer-och-informationsmaterial/, last accessed Oct 2016.

18. G. Gasser, M. Rona, A. Voloshenko, R. Shelkov, L. Tal, I. Pankratov, S. Elhanany, O. Lev, Quantitative Evaluation of Tracers for Quantification of Wastewater Contamination of Potable Water Sources, Environ. Sci. Technol., 2010, 44, 3919-3925.

19. A. W. Boorn, M.S. Cresser, R. F. Browner 1980. Evaporation characteristics of organic solvent aerosols used in analytical atomic spectrometry, Spectrochim. Acta Part B: At. Spec., $1980,35,823-832$.

20. L. Ebdon, M. Foulkes, K. Sutton, Slurry nebulization in plasmas, J. of anal. at spectrom., 1997, 12, 213-229.

21. S. Liu, D. Beauchemin, Effect of methanol and sodium dodecylsulfate on radial profiles of ion abundance in inductively coupled plasma mass spectrometry, Spectrochim. Acta Part B: At. Spect., 2006, 61, 319-325. 
22. L. E. Barton, M. Therezien, M. Auffan, J.Y. Bottero, M.R. Wiesner, Theory and Methodology for Determining Nanoparticle Affinity for Heteroaggregation in Environmental Matrices Using Batch Measurements, Environ. Eng. Sci., 2014, 31, 421-427.

23. B. Thalmann, A. Voegelin, B. Sinnet, E. Morgenroth, R. Kaegi, Sulfidation Kinetics of Silver Nanoparticles Reacted with Metal Sulfides, Environ. Sci. Tech., 2014, 48, 4885-4892.

24. R. D. Kent, J. G. Oser, P. J. Vikesland, Controlled evaluation of silver nanoparticle sulfidation in a full-scale wastewater treatment plant, Environ. Sci. Tech., 2014, 48, 8564-8572.

25. J. M. Pettibone, J. Liu, In Situ Methods for Monitoring Silver Nanoparticle Sulfidation in Simulated Waters, Environ. Sci. Tech., 2016, 50, 11145-11153.

26. M. Azodi, Y. Sultan, S. Ghoshal, Dissolution Behavior of Silver Nanoparticles and Formation of Secondary Silver Nanoparticles in Municipal Wastewater by Single Particle ICP-MS, Environ. Sci. Tech., Accepted for publication, DOI: 10.1021/acs.est.6b03957.

27. J. M. Wang, C.P. Huang, H. E. Allen, Surface physical-chemical characteristics of sludge particulates, Water Environ. Res., 2000, 72, 545-553.

28. C. L. Doolette, M. J. McLaughlin, J. K. Kirby, D. J. Batstone, H. H. Harris, H. Ge, G. Cornelis, Transformation of PVP coated silver nanoparticles in a simulated wastewater treatment process and the effect on microbial communities, Chem. Cent. J., 2013, 7:46,

29. S. H. Lin, M. R. Wiesner, Exact Analytical Expressions for the Potential of Electrical Double Layer Interactions for a Sphere-Plate System, Langmuir, 2010, 26, 16638-16641.

30. G. R. Wiese, T. W. Healy, Effect of particle size on colloid stability, Trans. Faraday Soc. 1970, 66, 490-499.

31. S. Ge, M. Agbakpe, W. Zhang, L. Kuang, Heteroaggregation between PEI-Coated Magnetic Nanoparticles and Algae: Effect of Particle Size on Algal Harvesting Efficiency, Acs Appl. Mat. \& Interf., 2015, 7, 6102-6108.

32.R. May, Y. S. Li, The effects of particle size on the deposition of fluorescent nanoparticles in porous media: Direct observation using laser scanning cytometry, Coll. Surf. A, 2013, 418, 84-91.

33. A. J. Pelley, N. Tufenkji, Effect of particle size and natural organic matter on the migration of nano- and microscale latex particles in saturated porous media, J. Coll. Interf. Sci., 2008, 321, 74-83.

34. G. Wulfsberg, Inorganic chemistry, University Science books, Sausalito CA, 2000.

35.J. Tuoriniemi, G. Cornelis, M. Hassellöv, Improving Accuracy of Single particle ICPMS for Measurement of Size Distributions and Number Concentrations of Nanoparticles by Determining Analyte Partitioning During Nebulisation, J. Anal. At. Spect., 2014, 29, 743-752. 
36. N.J. Miller-Ihli, Influence of slurry preparation on the accuracy of ultrasonic slurry electrothermal atomic absorption spectrometry, J. Anal. At. Spectrom., 1994, 9, 1129-1134.

37. J. C. Winterwerp, A simple model for turbulence induced flocculation of cohesive sediment, J. Hydraul. Res., 1998, 36, 309-326.

38. J. J. M. de Klein, J. T. K. Quik, P.S. Bäuerlein, A. A. Koelmans, Towards validation of the NanoDUFLOW nanoparticle fate model for the river Dommel, The Netherlands, environ. Sci. nano., 2016, 3, 434-441. 